Proyecciones

Vol. 21, No 2, pp. 155-174, August 2002.

Universidad Católica del Norte

Antofagasta - Chile

\title{
DIFFERENTIABILITY OF SOLUTIONS OF LINEAR FUNCTIONAL DIFFERENTIAL EQUATIONS WITH INFINITE DELAY
}

\author{
HERNÁN R. HENRÍQUEZ * \\ Universidad de Santiago, Chile
}

\begin{abstract}
In this note we establish a criterion to obtain classical solutions for a quasi-linear abstract retarded functional differential equation with infinite delay and we apply this result to characterize the infinitesimal generators of several strongly continuous semigroup of linear operators that arise in the theory of linear abstract retarded functional differential equations with infinite delay on a phase space defined axiomatically.
\end{abstract}

2000 Mathematics Subject Classification. $34 K 30,34 G 10$, $47 D 06$.

Key Words and Phrases. Abstract Retarded Functional Differential Equations ; Semigroup of linear operator ; Differentiability of Solutions.

*This work was supported by DICYT-USACH, Project 04-0133HM and FONDECYT, Project 1020259. 


\section{Introduction.}

In this work we are concerned with regularity properties of solutions of abstract retarded functional differential equations (abbreviated, ARFDE) with unbounded delay.

Let $X$ be a Banach space endowed with a norm $\|\cdot\|$. Throughout this paper we assume that $A: D(A) \rightarrow X$ is the infinitesimal generator of a strongly continuous semigroup of linear operators $T(t)$ defined on $X$. Our main purpose is to identify the domain of the infinitesimal generator of the solution semigroup corresponding to the initial value problem defined by the linear ARFDE with unbounded delay

$$
x^{\prime}(t)=A x(t)+L\left(x_{t}\right), \quad t \geq 0,
$$

with initial condition

$$
x_{0}=\varphi \in \mathcal{B},
$$

where $\mathcal{B}$ denotes an abstract phase space, $L: \mathcal{B} \rightarrow X$ is a bounded linear map and $x_{t}$ represents the function defined from $(-\infty, 0]$ into $X$ by $x_{t}(\theta)=x(t+\theta),-\infty<\theta \leq 0$.

Throughout this paper we will employ an axiomatic definition of the phase space $\mathcal{B}$ introduced by Hale and Kato [5]. To establish the axioms of space $\mathcal{B}$ we follow the terminology used in the book [9]. Thus, $\mathcal{B}$ will be a linear space of functions mapping $(-\infty, 0]$ into $X$ endowed with a seminorm $\|\cdot\|_{\mathcal{B}}$. We will assume that $\mathcal{B}$ satisfies the following axioms:

(A) If $x:(-\infty, \sigma+a) \rightarrow X, a>0$, is continuous on $[\sigma, \sigma+a)$ and $x_{\sigma} \in \mathcal{B}$ then for every $t$ in $[\sigma, \sigma+a)$ the following conditions hold:

(i) $x_{t}$ is in $\mathcal{B}$.

(ii) $\|x(t)\| \leq H\left\|x_{t}\right\|_{\mathcal{B}}$.

(iii) $\left\|x_{t}\right\|_{\mathcal{B}} \leq K(t-\sigma) \sup \{\|x(s)\|: \sigma \leq s \leq t\}+M(t-\sigma)\left\|x_{\sigma}\right\|_{\mathcal{B}}$, where $H \geq 0$ is a constant; $K, M:[0, \infty) \rightarrow[0, \infty), K$ is continuous and $M$ is locally bounded and $H, K$ and $M$ are independent of $x(\cdot)$. 
(A-1) For the function $x(\cdot)$ in $(A), x_{t}$ is a $\mathcal{B}$-valued continuous function on $[\sigma, \sigma+a)$.

(B) The space $\mathcal{B}$ is complete.

In the theory of retarded functional differential equations with unbounded delay frequently we need additional properties of the space $\mathcal{B}$ to obtain some results. Next we denote by $C_{00}$ the space of continuous functions from $(-\infty, 0]$ into $X$ with compact support. It is clear from the axioms of phase space that $C_{00} \subseteq \mathcal{B}$. In this work we consider the following axioms $([9])$.

(C-1) If $\left(\varphi^{n}\right)_{n}$ is a Cauchy sequence in $\mathcal{B}$ such that $\left(\varphi^{n}(\theta)\right)_{n}$ converges to a function $\varphi(\theta)$ uniformly on compact subsets of $(-\infty, 0]$, then $\varphi \in \mathcal{B}$ and $\left\|\varphi^{n}-\varphi\right\|_{\mathcal{B}} \rightarrow 0$ as $n \rightarrow \infty$.

(C-2) If a uniformly bounded sequence $\left(\varphi^{n}\right)_{n}$ in $C_{00}$ converges to a function $\varphi$ in the compact -open topology then $\varphi$ belongs to $\mathcal{B}$ and $\left\|\varphi^{n}-\varphi\right\|_{\mathcal{B}} \rightarrow 0$, as $n \rightarrow \infty$.

It is easy to see $([9])$ that if $(\mathrm{C}-2)$ holds then the space $C_{b}((-\infty, 0]$ : $X$ ) (or in short $C_{b}$ ) formed by the bounded continuous functions $\varphi$ : $(-\infty, 0] \rightarrow X$ is continuously included in $\mathcal{B}$. Hereafter we also use the notation $U C_{b}((-\infty, 0]: X)$ (abbreviated, $\left.U C_{b}\right)$ to indicate the subspace of $C_{b}$ formed by the functions uniformly continuous. Some aspects of our results depend on the following property of differentiability of functions with values in phase spaces .

Lemma 1.1 Assume that $\mathcal{B}$ satisfies axiom (C-2). Let $z:(-\infty, a) \rightarrow$ $X, a>0$, be a function of class $C^{1}$ such that $z_{0}=\varphi \in C_{b}$ and $\varphi^{\prime} \in U C_{b}$. Then the function $[0, a) \rightarrow \mathcal{B}, t \rightarrow z_{t}$, is continuosly differentiable and $\frac{d}{d t} z_{t}=z_{t}^{\prime}, 0 \leq t<a$.

Proof. It is clear that $z_{t}, z_{t}^{\prime} \in C_{b}, 0 \leq t<a$, and that for fixed $t<t_{1}<a$ the function $z^{\prime}$ is uniformly continuous on $\left(-\infty, t_{1}\right]$. This shows that $\left[0, t_{1}\right] \rightarrow C_{b}, t \rightarrow z_{t}^{\prime}$, is continuous. Hence, for $h$ small 
enough, from the theory of integration of vector functions with values in Banach spaces ([10]) we obtain that

$$
\begin{aligned}
\left(\int_{t}^{t+h} z_{s}^{\prime} d s\right)(\theta) & =\int_{t}^{t+h} z^{\prime}(s+\theta) d s \\
& =z(t+h+\theta)-z(t+\theta) \\
& =z_{t+h}(\theta)-z_{t}(\theta)
\end{aligned}
$$

which implies that

$$
\frac{z_{t+h}-z_{t}}{h}=\frac{1}{h} \int_{t}^{t+h} z_{s}^{\prime} d s
$$

converges to $z_{t}^{\prime}$ as $h \rightarrow 0$.

Throughout this paper we always assume that $\mathcal{B}$ is a phase space. We will denote by $\widehat{\mathcal{B}}$ the quotient Banach space $\mathcal{B} /\|\cdot\|$ and we write $\hat{\varphi}$ for the coset determined by $\varphi \in \mathcal{B}$. We also use this notation to indicate induced maps on $\widehat{\mathcal{B}}$ whilst the symbol $\pi$ will stand for the quotient map from $\mathcal{B}$ into $\widehat{\mathcal{B}}$.

It is follows from the axioms of phase space that operators $S(t)$ and $W(t)$ defined by the expressions

$$
[S(t) \varphi](\theta):=\left\{\begin{aligned}
\varphi(0), & -t \leq \theta \leq 0, \\
\varphi(t+\theta), & -\infty<\theta<-t,
\end{aligned}\right.
$$

and

$$
[W(t) \varphi](\theta):=\left\{\begin{aligned}
T(t+\theta) \varphi(0), & -t \leq \theta \leq 0, \\
\varphi(t+\theta), & -\infty<\theta<-t .
\end{aligned}\right.
$$

are strongly continuous semigroups of linear operators on $\mathcal{B}$.

On the other hand, the solution operator of problem (1.1)-(1.2) given by $V(t) \varphi=x_{t}(\cdot, \varphi)$, where $x(\cdot, \varphi)$ denotes the mild solution of (1.1)-(1.2), also is a strongly continuous semigroup on $\mathcal{B}$.

For the general aspects of the theory of strongly continuous semigroup of linear operators we refer to Pazy [11] and Engel and Nagel [3]. In particular, $\widehat{S}(t), \widehat{W}(t)$ and $\widehat{V}(t)$ are strongly continuous semigroups of linear operators on the Banach space $\widehat{\mathcal{B}}$. Their infinitesimal generators will be denoted by $\widehat{B}, \widehat{B_{W}}$ and $\widehat{A_{V}}$, respectively. 
For some concrete phase spaces the domain of operators $\widehat{B}, \widehat{B_{W}}$ and $\widehat{A_{V}}$ is well known $([9,6,12])$. Nevertheless, a general characterization in the frame of phase spaces defined axiomatically is unknown. The purpose of this work is, under quite general conditions on $X$ and $\mathcal{B}$, to obtain some relations among these domains. To establish our results we need some additional properties of differentiability of functions. For completeness we state them here.

For Banach spaces that have the Radon-Nikodym property (abbreviated, RNP) the following result is well known ([2]).

Lemma 1.2 Let $X$ be a Banach space which has the RNP. If a function $f:[0, a] \rightarrow X$ is Lipschitz continuous then $f$ is differentiable a.e. and $f(t)=f(0)+\int_{0}^{t} f^{\prime}(s) d s$, for $0 \leq t \leq a$.

We can modify easily this result to work with semi-normed spaces.

Corollary 1.1 Let $Y$ be a complete semi-normed space such that the quotient space $Y /\|\cdot\|$ has the RNP. If a function $f:[0, a] \rightarrow Y$ is Lipschitz continuous then $f$ is differentiable a.e. and $\| f(t)-f(0)-$ $\int_{0}^{t} f^{\prime}(s) d s \|=0$, for $0 \leq t \leq a$.

Furthermore, the following statement is an easy consequence of Lemma 1.2 and properties of differentiability of semigroups .

Remark 1.1 Assume that $X$ is a Banach space which has the RNP and that $T(\cdot) x$ is locally Lipschitz continuous . Then $T(\cdot) x$ is differentiable on $(0, \infty)$.

On the other hand, since $X$ can be considered as a closed subspace of $\mathcal{B}$, if $\widehat{\mathcal{B}}$ has the RNP then $X$ also has this property.

Our results are based on the following property of regularity for mild solutions of the abstract Cauchy problem which was established in $([4])$.

Lemma 1.3 Assume that $X$ is a Banach space which has the RNP and that $f$ is a function that satisfies the Lipschitz condition

$$
\|f(s, x)-f(t, y)\| \leq C(|t-s|+\|x-y\|) .
$$


Let $x(\cdot)$ be the mild solution of the abstract Cauchy problem

$$
x^{\prime}(t)=A x(t)+f(t, x(t)), \quad 0 \leq t \leq a, \quad x(0) \in D(A),
$$

then $x \in C^{1}([0, a] ; X)$.

In the next section we generalize the above result for ARFDE with unbounded delay and in section 3 we apply our results to the linear case.

\section{Regularity of mild solutions of ARFDE.}

In this section we present a result of regularity for mild solutions of quasi-linear ARFDE with unbounded delay

$$
x^{\prime}(t)=A x(t)+f\left(t, x_{t}\right), \quad 0 \leq t<a,
$$

where $f:[0, a) \times \mathcal{B} \rightarrow X, a>0$, is a continuous function.

We consider the following concept of mild solution.

Definition 2.1 We say that a function $x:(-\infty, b) \rightarrow X, b>0$, is a mild solution of the Cauchy problem (2.1)-(1.2) if $x_{0}=\varphi$ and the restriction $x:[0, b) \rightarrow X$ is continuous and satisfies the integral equation:

$$
x(t)=T(t) \varphi(0)+\int_{0}^{t} T(t-s) f\left(s, x_{s}\right) d s, \quad 0 \leq t<b .
$$

The problem of existence of mild solutions for equation (2.1) it has been studied in $[7,8]$ under several conditions on $T(t)$ and $f$. In particular, we can assure existence of mild solutions when $f$ is locally Lipschitz continuous. In the next result we denote by $x(\cdot, \varphi)$ the mild solution of problem (2.1)-(1.2). We also refer the reader to [12, 13], as well as the references indicated therein, for the nonlinear analogue.

Theorem 2.1 Assume that $X$ has the RNP and that $W(\cdot) \varphi$ is locally Lipschitz continuous. If $f$ satisfies the Lipschitz condition:

$$
\left\|f\left(s, \psi^{1}\right)-f\left(t, \psi^{2}\right)\right\| \leq C\left(|t-s|+\left\|\psi^{1}-\psi^{2}\right\|_{\mathcal{B}}\right),
$$

for some constants $C, r>0$ and for every $s, t \in[0, r)$ and $\left\|\psi^{i}-\varphi\right\|_{\mathcal{B}}<r, i=1,2$, then the following properties are fulfilled : 
(i) The function $x(\cdot, \varphi)$ is of class $C^{1}$ on $(0, b)$, for some $b>0$, and if $\varphi(0) \in D(A)$, then $x(\cdot, \varphi)$ is of class $C^{1}$ on $[0, b)$.

(ii) If $X$ is reflexive then $x(\cdot, \varphi)$ is of class $C^{1}$ on $[0, b)$.

(iii) If $\widehat{\mathcal{B}}$ has the RNP then the function $t \rightarrow x_{t}(\cdot, \varphi)$ is differentiable a.e. on $[0, b)$.

(iv) If $\mathcal{B}$ satisfies axiom (C-2), $f(0, \varphi)=0$ and $W(\cdot) \varphi$ is differentiable on $(0, \infty)$ (resp. on $[0, \infty))$ then the function $t \rightarrow x_{t}(\cdot, \varphi)$ is differentiable on $(0, b)$ (resp. on $[0, b)$ ).

Proof. In view of previous remark we admit the existence of the mild solution $x(\cdot, \varphi)$. Next we abbreviate the notation by writing $x=x(\cdot, \varphi)$. We introduce the functions $y, z:(-\infty, b) \rightarrow X$ where $y(\cdot)$ is defined by $y(t)=T(t) \varphi(0)$, for $t \geq 0$, and $y(t)=\varphi(t)$, for $t<0$, and $z(\cdot)$ is given by the relation

$$
x(t)=y(t)+z(t), \quad-\infty<t<b .
$$

It is clear from these definitions and (2.2) that $y_{t}=W(t) \varphi, \quad z_{0}=0$ and

$$
z(t)=\int_{0}^{t} T(t-s) f\left(s, x_{s}\right) d s, \quad 0 \leq t<b .
$$

Proceeding as in ([6], Theorem 2) we can show that $z$ is Lipschitz continuous. Since $z_{t}$ is continuous with compact support, it follows from the axioms of phase space that the map $t \rightarrow z_{t}$ also is Lipschitz continuous . Moreover, since expression (2.4) yields

$$
x_{t}=y_{t}+z_{t}=W(t) \varphi+z_{t}, \quad 0 \leq t<b .
$$

we obtain that $x_{t}$ also verifies a Lipschitz condition which, together with (2.3), imply that the function $t \rightarrow f\left(t, x_{t}\right)$ is Lipschitz continuous. Applying Lemma 1.2 and Theorem 1 in [4] we derive that $z \in C^{1}([0, b): X)$. On the other hand, from the axiom (A-ii) we know that

$$
\|T(t) \varphi(0)-T(s) \varphi(0)\| \leq H\|W(t) \varphi-W(s) \varphi\|_{\mathcal{B}}
$$


Thus, from Remark 1.1 it follows that $T(\cdot) \varphi(0)$ is differentiable on $(0, \infty)$ and the first part of assertion (i) is immediate consequence of (2.2). Similarly, if $\varphi(0) \in D(A)$, then $T(\cdot) \varphi(0)$ is differentiable on $[0, \infty)$ and the same occurs with $x(\cdot)$.

To establish (ii) we turn to use that $T(\cdot) \varphi(0)$ is a function Lipschitz continuous . Since $X$ is reflexive, from [1]. Theorem 2.1.2(c), it follows that $\varphi(0) \in D(A)$ so that the assertion is consequence of (i).

Since $t \rightarrow x_{t}$ is Lipschitz continuous, the assertion (iii) is an immediate consequence of Corollary 1.1.

Finally, if $f(0, \varphi)=0$ the function $z(\cdot)$ is of class $C^{1}$ on $(-\infty, b)$. In fact, in view of that we already have established, only remains to prove that $z^{\prime}(0)=0$. However, this is a direct consequence of (2.5). Hence, by Lemma 1.1 we can affirm that the function $t \rightarrow z_{t}$ is differentiable on $[0, b)$ and the equality (2.6) implies assertion (iv).

Related with this result some observations are useful. First, we notice that under the conditions of the theorem $x(t, \varphi) \in D(A)$ and equation (2.1) is verified for $t>0$ ([11], Theorem 2.4). On the other hand, the possible applications of the theorem depend on the hypothesis that the function $W(\cdot) \varphi$ is locally Lipschitz continuous. Next we establish some conditions to obtain such property.

Proposition 2.1 If $T(\cdot) \varphi(0)$ and $S(\cdot) \varphi$ are locally Lipschitz continuous then $W(\cdot) \varphi$ is also locally Lipschitz continuous.

Proof. We define $R(t)=W(t)-S(t)$. It is clear from the definitions of $S$ and $W$ that $R(t) \varphi$ is a continuous function with compact support included in $[-t, 0]$. Therefore, for $a>0$ fixed and $0 \leq s \leq t \leq a$ the function $R(t) \varphi-R(s) \varphi$ is a continuous function with compact support included in $[-a, 0]$. Consequently, axiom (A-iii) implies that

$$
\begin{array}{r}
\|R(t) \varphi-R(s) \varphi\|_{\mathcal{B}} \leq K_{a} \max \left\{\max _{-t \leq \theta \leq-s}\|T(t+\theta) \varphi(0)-\varphi(0)\|,\right. \\
\left.\max _{-s \leq \theta \leq 0}\|T(t+\theta) \varphi(0)-T(s+\theta) \varphi(0)\|\right\},
\end{array}
$$

where $K_{a}=\max _{0 \leq t \leq a} K(t)$. Since $T(\cdot) \varphi(0)$ is locally Lipschitz continuous 
the above estimate shows that $R(\cdot) \varphi$ is also locally Lipschitz continuous which in turn implies that $W(t) \varphi=R(t) \varphi+S(t) \varphi$ has the same property.

We can give a condition to obtain $S(\cdot) \varphi$ to be Lipschitz continuous.

Proposition 2.2 Assume that $\mathcal{B}$ satisfies $(\mathrm{C}-2)$ and let $\varphi \in C_{b}((-\infty, 0] ; X)$.

(a) If $\varphi$ is a function uniformly Lipschitz continuous then $S(\cdot) \varphi$ is locally Lipschitz continuous .

(b) If $\widehat{\mathcal{B}}$ is reflexive and if $\varphi$ is a function uniformly Lipschitz continuous then $\widehat{\varphi} \in D(\widehat{B})$.

(c) If $\varphi$ is a function of class $C^{1}$ such that $\varphi^{\prime} \in U C_{b}$ and $\varphi^{\prime}(0)=0$, then $\widehat{\varphi} \in D(\widehat{B})$ and $\widehat{B} \widehat{\varphi}=\widehat{\varphi^{\prime}}$.

Proof. Since $C_{b} \hookrightarrow \mathcal{B}$ and $S(\cdot)$ is uniformly bounded on bounded intervals the assertion (a) follows from the estimate

$$
\begin{aligned}
& \|S(t) \varphi-\varphi\|_{\infty}=\sup _{\theta \leq 0}\|[S(t) \varphi](\theta)-\varphi(\theta)\| \\
& \leq \max \left\{\max _{-t \leq \theta \leq 0}\|\varphi(\theta)-\varphi(\theta)\|, \sup _{\theta \leq-t}\|\varphi(t+\theta)-\varphi(\theta)\|\right\} \\
& \leq C t,
\end{aligned}
$$

for some constant $C \geq 0$ that only depends on $\varphi$.

The assertion (b) is an immediate consequence of (a) and Theorem 2.1.2(c) in [1].

To prove (c) we define the function $x: \mathbb{R} \rightarrow X$ by $x(\theta)=$ $\varphi(\theta),-\infty<\theta \leq 0$, and $x(t)=\varphi(0), t>0$. It is clear that $x$ is differentiable and $x^{\prime}$ is uniformly continuous and bounded on $\mathbb{R}$. By Lemma 1.1 the function $t \rightarrow x_{t}=S(t) \varphi$ is differentiable on $[0, \infty)$ and

$$
\frac{d}{d t} \widehat{S}(t) \widehat{\varphi}=\widehat{x_{t}^{\prime}}
$$

In particular, evaluating the derivative at $t=0$ we obtain

$$
\widehat{B} \widehat{\varphi}=\left.\frac{d}{d t} \widehat{S}(t) \widehat{\varphi}\right|_{t=0}=\widehat{x_{0}^{\prime}}=\widehat{\varphi^{\prime}}
$$




\section{Applications to linear ARFDE.}

In this section we apply Theorem 2.1 to study differentiability of mild solutions of the linear inhomogeneous ARFDE with unbounded delay

$$
x^{\prime}(t)=A x(t)+L\left(x_{t}\right)+h(t), \quad t \geq 0,
$$

where $L: \mathcal{B} \rightarrow X$ is a bounded linear map and $h:[0, \infty) \rightarrow X$ is locally integrable. The mild solution of (3.1)-(1.2), denoted $x(\cdot, \varphi, h)$, is defined on $\mathbb{R}$ and satisfies

$$
x(\cdot, \varphi, h)=x(\cdot, \varphi, 0)+x(\cdot, 0, h) .
$$

Initially we consider the homogeneous linear problem (1.1)-(1.2). In this case $V(t) \varphi=x_{t}(\cdot, \varphi, 0), t \geq 0$, is the solution semigroup . With the notations introduced in the proof of Theorem 2.1 we can rewrite expression (2.6) as

$$
V(t) \varphi=W(t) \varphi+z_{t}, \quad t \geq 0
$$

In [6] we have introduced the following additional axiom for phase spaces .

(C-3) Let $a>0$. Let $x:(-\infty, a] \rightarrow X$ be a continuous function such that $x_{0}=0$ and the right derivative at 0 , denoted $x_{R}^{\prime}(0)$, exists. If the function $\psi$ defined by $\psi(\theta)=0$, for $\theta<0$, and $\psi(0)=x_{R}^{\prime}(0)$ belongs to $\mathcal{B}$, then $\left\|\frac{1}{h} x_{h}-\psi\right\|_{\mathcal{B}} \rightarrow 0$, as $h \rightarrow 0^{+}$.

We refer the reader to [6] for examples of phase spaces that satisfy this axiom.

Using (3.3) and proceeding as in theorems 3 and 4 in [6] we can prove easily that if one of the following conditions holds:

(i) $\mathcal{B}$ satisfies axiom $(\mathrm{C}-3)$ and the function $\Gamma$ defined by $\Gamma(\theta)=$ $0,-\infty<\theta<0$, and $\Gamma(0)=L(\varphi)$ belongs to $\mathcal{B}$;

(ii) $L(\varphi)=0$, 
then $\varphi \in D\left(B_{W}\right)$ if, and only if, $\varphi \in D\left(A_{V}\right)$. In fact, in view of (3.3) to establish the assertion we only need to show that $\frac{1}{t} z_{t}$ is convergent as $t \rightarrow 0^{+}$. From $(2.5)$ we can write

$$
z(t)=\int_{0}^{t} T(t-s) L(V(s) \varphi) d s, \quad t \geq 0 .
$$

If we assume that (i) holds, the above expression easily implies that $\frac{1}{t} z_{t} \rightarrow \Gamma$, as $t \rightarrow 0^{+}$. On the other hand, from the expression for $z$ we obtain the estimation

$$
\left\|\frac{1}{t} z_{t}\right\|_{\mathcal{B}} \leq \frac{1}{t} K(t) \max _{-t \leq \theta \leq 0}\left\|\int_{0}^{t+\theta} T(t+\theta-s) L(V(s) \varphi) d s\right\| .
$$

Hence, if (ii) is verified then clearly $\frac{1}{t} z_{t} \rightarrow 0, t \rightarrow 0^{+}$.

In our next result we consider a different and, in some sense, a more general condition to relate the differentiability of $V(\cdot) \varphi$ and $W(\cdot) \varphi$. As consequence we obtain a new result to compare the domains of operators $\widehat{A_{V}}$ and $\widehat{B_{W}}$.

Theorem 3.1 Assume that $X$ has the RNP and let $\varphi \in \mathcal{B}$ such that $W(\cdot) \varphi$ is locally Lipschitz continuous . Then the following properties are fulfilled :

(a) The mild solution $x(\cdot, \varphi, 0)$ is a function of class $C^{1}$ on $(0, \infty)$ and $V(\cdot) \varphi$ is locally Lipschitz continuous . If, in addition, $\varphi(0) \in$ $D(A)$ then $x(\cdot, \varphi, 0)$ is a function of class $C^{1}$ on $[0, \infty)$.

(b) If $\widehat{\mathcal{B}}$ has the RNP then $V(\cdot) \varphi$ is differentiable on $(0, \infty)$.

Proof. The assertion (a) follows directly from Theorem 2.1. To prove (b), we observe that Theorem 2.1 ensures that $V(\cdot) \varphi$ is differentiable a.e. on $[0, \infty)$. Since $V(\cdot)$ is a strongly continuous semigroup from Remark 1.1 we obtain the differentiability of $V(\cdot) \varphi$ on $(0, \infty)$. 
Corollary 3.1 If $\widehat{\mathcal{B}}$ is reflexive then $D\left(\widehat{A_{V}}\right)=D\left(\widehat{B_{W}}\right)$.

Proof. If $\widehat{\varphi} \in D\left(\widehat{B_{W}}\right)$, then $W(\cdot) \varphi$ is continuosly differentiable and by part (a) of Theorem 1 it follows that $V(\cdot) \varphi$ is locally Lipschitz continuous . since $\widehat{\mathcal{B}}$ is reflexive from Theorem 2.1.2(c) in ([1]) we obtain that $\hat{\varphi} \in D\left(\widehat{A_{V}}\right)$.

Reciprocally, if $\hat{\varphi} \in D\left(\widehat{A_{V}}\right)$, then $V(\cdot) \varphi$ is continuosly differentiable and Lipschitz continuous. From (3.4) it follows easily that $z(\cdot)$ is Lipschitz continuous. Proceeding as in the proof of Theorem 2.1 we conclude that the function $t \rightarrow z_{t}$ is also Lipschitz continuous . Applying now (3.3) we obtain that the same occurs with $W(\cdot) \varphi$ so that we can complete the proof arguing as before.

This result is similar in nature to Theorem 5.5.6 in [9] which establishes, for $X$ finite dimensional, that $D\left(A_{V}^{*}\right)=D\left(B^{*}\right)$. Thus, in this case, the domain of adjoint operator $A_{V}^{*}$ is independent of $L$. Turning now to consider the general abstract case, we can assert, at least in the case $\widehat{\mathcal{B}}$ reflexive, that $D\left(\hat{A_{V}}\right)$ is independent of $L$. Furthermore, if $\widehat{\varphi} \in D\left(\widehat{B_{W}}\right)$, from (3.3) we infer that

$$
\begin{aligned}
{\left[\widehat{A_{V}} \hat{\varphi}\right](0) } & =\left[\widehat{B_{W}} \hat{\varphi}\right](0)+L(\varphi) \\
& =A \varphi(0)+L(\varphi) .
\end{aligned}
$$

From these results arise the importance to characterize $D\left(\widehat{B_{W}}\right)$ in order to know $D\left(\widehat{A_{V}}\right)$. Our next proposition gives some conditions to guarantee that an element $\hat{\varphi}$ belongs to $D\left(\widehat{(B}_{W}\right)$.

Proposition 3.1 Assume that $\mathcal{B}$ satisfies (C-2) and let $\varphi \in$ $C_{b}((-\infty, 0] ; X)$ be a function uniformly Lipschitz continuous such that $\varphi(0) \in D(A)$.

(a) If $\widehat{\mathcal{B}}$ has the RNP then $\widehat{W}(t) \widehat{\varphi} \in D\left(\widehat{B_{W}}\right), t>0$.

(b) If $\widehat{\mathcal{B}}$ is reflexive then $\widehat{\varphi} \in D\left(\widehat{B_{W}}\right)$.

The proof will be omitted because is similar to that carried out in Proposition 2.2. 
Now we are going to study the differentiability of mild solutions of the inhomogeneous equation (3.1). By the decomposition (3.2) we can restrict us to consider the initial condition $\varphi=0$.

Related with the semigroup $T$ we introduce a class $\mathcal{L}$ of functions defined as follows. We say that a function $h:[0, \infty) \rightarrow X$ belongs to $\mathcal{L}$ if $h$ is locally integrable, locally bounded and for each $a>0$ there exists a constant $C_{a} \geq 0$ such that

$$
\left\|\left[T\left(t_{2}\right)-T\left(t_{1}\right)\right] h(s)\right\| \leq C\left|t_{2}-t_{1}\right|,
$$

for all $0 \leq s, t_{1}, t_{2} \leq a$.

Lemma 3.1 Assume that $X$ has the RNP. Let $h \in \mathcal{L}$ be a continuous function. Then for each $t \geq 0$ the function $s \rightarrow A T(t-s) h(s)$ is integrable on $[0, t)$ and the function $u$ defined by

$$
u(t)=\int_{0}^{t} T(t-s) h(s) d s, \quad t \geq 0,
$$

is of class $C^{1}$ with

$$
u^{\prime}(t)=h(t)+\int_{0}^{t} A T(t-s) h(s) d s, \quad t \geq 0 .
$$

Proof. Since $T(\cdot) h(s)$ is locally Lipschitz continuous from Remark 1.1 we have that $T(\cdot) h(s)$ is differentiable on $(0, \infty)$ which implies that $T(t) h(s) \in D(A)$, for all $t>0$. Now, we take $t>0$ fixed and consider the functions

$$
g_{\alpha}(s)=\frac{T(\alpha+t-s) h(s)-T(t-s) h(s)}{\alpha}, \alpha>0,
$$

defined for $s \in[0, t)$. It is clear that $g_{\alpha}(s) \rightarrow A T(t-s) h(s)$, as $\alpha \rightarrow 0^{+}$and, since $h \in \mathcal{L},\left\|g_{\alpha}(s)\right\| \leq C$, for all $s \in[0, t)$ and certain constant $C \geq 0$. By the Lebesgue's dominated convergence theorem ([10]) we conclude that $s \rightarrow A T(t-s) h(s)$ is integrable on $[0, t)$. We complete the proof applying Theorem 4.2.4 in [11].

We are in conditions to establish the following regularity property for mild solutions of the inhomogeneous ARFDE. 
Theorem 3.2 Assume that $X$ has the RNP. If $h$ satisfies one of the following conditions:

(a) $h$ is locally Lipschitz continuous ;

(b) $h \in \mathcal{L}$ is a continuous function

then $x(\cdot, 0, h)$ is of class $C^{1}$ on $[0, \infty)$ and the following properties hold:

(i) If $\widehat{\mathcal{B}}$ has the $\mathrm{RNP}$ then $x_{t}(\cdot, 0, h)$ is differentiable a.e. on $[0, \infty)$.

(ii) If $\mathcal{B}$ satisfies axiom $(\mathrm{C}-2)$ and $h(0)=0$ then the function $t \rightarrow x_{t}(\cdot, 0, h)$ is differentiable on $[0, \infty)$.

Proof. If condition (a) holds then the assertions are consequence of Theorem 2.1 with $f(t, \psi)=L(\psi)+h(t)$ and $\varphi=0$. For this reason in what follows we assume that $h$ satisfies condition (b). We abbreviate the notation by writing $x=x(\cdot, 0, h)$. Since

$$
x(t)=\int_{0}^{t} T(t-s) L\left(x_{s}\right) d s+\int_{0}^{t} T(t-s) h(s) d s
$$

we can write

$$
x(t)=z(t)+u(t), \quad t \in \mathbb{R},
$$

where $u$ is defined by (3.5) for $t \geq 0$ and $u(\theta)=0$, for $\theta \leq 0$ and $z$ is given by the above expression. This decomposition implies that

$$
x_{t}=z_{t}+u_{t}, \quad t \geq 0 .
$$

On the other hand, by Lemma 1 we know that $u$ is locally Lipschitz continuous on $[0, \infty)$ and since $u_{t}$ are continuous functions with compact support, applying axiom (A-iii) we obtain that both the function $t \rightarrow u_{t}$ as the function $v$ which is given by

$$
v(t)=\int_{0}^{t} T(t-s) L\left(u_{s}\right) d s
$$


are also locally Lipschitz continuous on $[0, \infty)$.

In view of that

$$
z(t)=\int_{0}^{t} T(t-s) L\left(z_{s}\right) d s+v(t), \quad t \geq 0
$$

with the argument used in ([6], Theorem 2) we infer that $z$ is locally Lipschitz continuous on $[0, \infty)$. Now repeating the argument used in the proof of Theorem 2.1 we obtain that $z$ is of class $C^{1}$ on $[0, \infty)$. Since by Lemma 1 this is also valid for $u$ it follows that $x$ is of class $C^{1}$ on $[0, \infty)$. The assertion (i) is consequence of Lemma 2. Finally, if the hypotheses of (ii) are verified, from Lemma 3.1 and Lemma 1 we obtain that the functions $t \rightarrow z_{t}$ and $t \rightarrow u_{t}$ are differentiable, which completes the proof.

Collecting theorems 3.1, 3.2 and the formula (3.2) we obtain the following property of differentiability for the solutions of (3.1)-(1.2).

Corollary 3.2 Assume that $X$ has the $\operatorname{RNP}$ and let $\varphi \in \mathcal{B}$ such that $W(\cdot) \varphi$ is locally Lipschitz continuous. If $h$ satisfies one of the following conditions:

(a) $h$ is locally Lipschitz continuous ;

(b) $h \in \mathcal{L}$ is a continuous function,

then $x(\cdot, \varphi, h)$ is of class $C^{1}$ on $(0, \infty)$. In further, if $\varphi(0) \in D(A)$, then $x(\cdot, \varphi, h)$ is of class $C^{1}$ on $[0, \infty)$.

The argument used in the proof of Theorem 3.2 allows us to generalize to the context of ARFDE with infinite delay the characterization of the infinitesimal generator of the solution semigroup obtained in [14] for ARFDE with finite delay modelled in the space of continuous functions . Previously we introduce a separation axiom for the phase spaces .

(S) If $\varphi \in \mathcal{B}$ is a continuous function such that $\|\varphi\|_{\mathcal{B}}=0$, then $\varphi=0$.

Next we establish a property of differentiability in phase spaces. 
Lemma 3.2 Assume that $\mathcal{B}$ satisfies axioms (C-1) and (S). Let $x$ : $(-\infty, b] \rightarrow X, b>0$, be a continuous function such that $x_{0} \in \mathcal{B}$. Then

$$
\left(\int_{0}^{b} x_{s} d s\right)(\theta)=\int_{0}^{b} x(s+\theta) d s, \quad-\infty<\theta \leq 0 .
$$

Proof. Let $\psi^{n}=\sum_{i=1}^{k(n)} \frac{b}{k(n)} x_{s_{i}}$ be a Riemann sum of $x_{s}$, where $s_{i}=\frac{b}{k(n)} i, \quad i=1, \cdots, k(n), \quad$ and $k(n) \rightarrow \infty \quad$ as $n \rightarrow \infty$. Then $\psi^{n} \rightarrow \int_{0}^{b} x_{s} d s$ as $n \rightarrow \infty$ in the space $\mathcal{B}$. Furthermore, for each $-\infty<\theta \leq 0$,

$$
\psi^{n}(\theta)=\frac{b}{k(n)} \sum_{i=1}^{n} x_{s_{i}}(\theta)=\frac{b}{k(n)} \sum_{i=1}^{k(n)} x\left(s_{i}+\theta\right)
$$

is in turn a Riemann sum for the function $x(s+\theta)$ on the interval $[0, b]$. Consequently,

$$
\psi^{n}(\theta) \rightarrow \int_{0}^{b} x(s+\theta) d s, n \rightarrow \infty
$$

On the other hand, since $x(\cdot)$ is uniformly continuous on bounded intervals, the last convergence is uniform for $\theta$ in a bounded interval. Thus, if we define $\psi$ by

$$
\psi(\theta)=\int_{0}^{b} x(s+\theta) d s
$$

we have shown that $\psi^{n} \rightarrow \psi, n \rightarrow \infty$, in the compact-open topology. Applying axiom (C-1) we can assert that $\psi^{n} \rightarrow \psi, n \rightarrow \infty$, in the space $\mathcal{B}$. Hence it follows that $\left\|\psi-\int_{0}^{b} x_{s} d s\right\|_{\mathcal{B}}=0$, which by the separation axiom implies that

$$
\psi(\theta)=\int_{0}^{b} x(s+\theta) d s=\left(\int_{0}^{b} x_{s} d s\right)(\theta) .
$$


Lemma 3.3 Assume that $\mathcal{B}$ satisfies axioms $(\mathrm{C}-1)$ and $(\mathrm{S})$. Let $z$ : $(-\infty, a) \rightarrow X, a>0$, be a function of class $C^{1}$ such that $z_{0}=\varphi \in \mathcal{B}$ and $\varphi^{\prime} \in \mathcal{B}$. Then the function $t \rightarrow z_{t}(\cdot)$ is of class $C^{1}$ on $[0, a)$ and $\frac{d}{d t} z_{t}=z_{t}^{\prime}$, for $t \in[0, a)$.

Proof. Because $z^{\prime}$ is continuous an application of the previous lemma yields

$$
\left(\int_{0}^{t} z_{s}^{\prime} d s\right)(\theta)=\int_{0}^{t} z^{\prime}(s+\theta) d s=z(t+\theta)-z(\theta)
$$

for each $\theta \leq 0$. Therefore, $\int_{0}^{t} z_{s}^{\prime} d s=z_{t}-z_{0}, 0 \leq t<a$, from which we obtain easily our result.

Now we are in conditions to establish the following characterization of $D\left(\widehat{A_{V}}\right)$.

Proposition 3.2 Let $\varphi \in \mathcal{B}$ be a function of class $C^{1}$ such that $\varphi^{\prime} \in \mathcal{B}, \varphi(0) \in D(A)$ and $\varphi^{\prime}(0)=A \varphi(0)+L(\varphi)$. If one of the following conditions is fulfilled :

(a) The space $\mathcal{B}$ satisfies $(\mathrm{C}-2)$ and $\varphi \in C_{b}((-\infty, 0] ; X), \varphi^{\prime} \in U C_{b}$;

(b) The space $\mathcal{B}$ satisfies (C-1) and (S).

Then $\widehat{\varphi} \in D\left(\widehat{A_{V}}\right)$ and $\widehat{A_{V}} \widehat{\varphi}=\widehat{\varphi^{\prime}}$.

Proof. Let $x=x(\cdot, \varphi)$ be the mild solution of the homogeneous problem (1.1)-(1.2). Proceeding as before we can decompose

$$
x(t)=z(t)+u(t), \quad t \in \mathbb{R},
$$

where we have modified our previous notations by defining $z_{0}=0, u_{0}=$ $\varphi$ and

$$
\begin{aligned}
& z(t)=\int_{0}^{t} T(t-s) L(V(s) \varphi-\varphi) d s, \\
& u(t)=T(t) \varphi(0)+\int_{0}^{t} T(s) L(\varphi) d s,
\end{aligned}
$$


for $t \geq 0$. Since $V(s) \varphi-\varphi \rightarrow 0$, as $s \rightarrow 0$, a simple estimation gives that $\frac{z_{t}}{t} \rightarrow 0$, as $t \rightarrow 0^{+}$. Furthermore, $u$ is of class $C^{1}$ on $[0, \infty)$ and $u^{\prime}(0)=A \varphi(0)+L(\varphi)=\varphi^{\prime}(0)$, which shows that $u$ is of class $C^{1}$ on IR.

In the case (a), from Lemma 1.1 we infer that $t \rightarrow u_{t}$ is differentiable and using relation (3.6) we conclude that $t \rightarrow x_{t}=V(t) \varphi$ is differentiable at $t=0$. Moreover, $\widehat{A_{V}} \widehat{\varphi}=\widehat{V}^{\prime}(0) \hat{\varphi}=\widehat{u}_{0}^{\prime}=\widehat{\varphi^{\prime}}$. The proof in case (b) is similar, but using Lemma 3.3 instead of Lemma 1.1.

It is clear that if $L=0$, then $V=W$. Consequently, taking $L=0$ we obtain the following result, which generalizes Proposition 2.2(c).

Corollary 3.3 Let $\varphi \in \mathcal{B}$ be a function of class $C^{1}$ such that $\varphi^{\prime} \in$ $\mathcal{B}, \varphi(0) \in D(A)$ and $\varphi^{\prime}(0)=A \varphi(0)$. If one of the following conditions is fulfilled :

(a) The space $\mathcal{B}$ satisfies $(\mathrm{C}-2)$ and $\varphi \in C_{b}((-\infty, 0] ; X), \varphi^{\prime} \in U C_{b}$;

(b) The space $\mathcal{B}$ satisfies (C-1) and (S).

Then $\widehat{\varphi} \in D\left(\widehat{B_{W}}\right)$ and $\widehat{B_{W}} \widehat{\varphi}=\widehat{\varphi^{\prime}}$.

Finally, it should be pointed out that there are phase spaces that satisfy the assumptions considered in our results. As an example we only mention the space $\mathcal{B}=C_{0} \times L^{p}(g)$, with the terminology of [9], which consists of all functions $\varphi:(-\infty, 0] \rightarrow X$ such that $\varphi$ is Lebesgue-measurable and $g\|\varphi\|^{p}$ is Lebesgue integrable on $(-\infty, 0)$, where $g:(-\infty, 0) \rightarrow \mathbb{R}$ is a positive Lebesgue integrable function (see Marle [10] for concepts relatives to the integration of vector functions). The seminorm in $\mathcal{B}$ is defined by

$$
\|\varphi\|=\|\varphi(0)\|+\left(\int_{-\infty}^{0} g(\theta)\|\varphi(\theta)\|^{p} d \theta\right)^{1 / p} .
$$

We will assume that $g$ satisfies conditions (g-6) and (g-7) in the terminology of [9]. In this case, $\mathcal{B}$ is a phase space which verifies axioms (A), (A-1), (B) and (C-2) ([9], Theorem 1.3.8). Furthermore, if $1<p<\infty$ and $X$ has the RNP (resp. is reflexive) then $\mathcal{B}$ also has the RNP (resp. is reflexive). 


\section{References}

[1] P. L. Butzer and H. Berens, Semi-Groups of Operators and Approximation. Springer-Verlag, Berlin, (1967).

[2] J. Diestel and J. J. Uhl, Vector Measures. Amer. Math. Society, (1972).

[3] Engel, K-J. and R. Nagel, One-parameter Semigroups for Linear Evolution Equations. Springer-Verlag, New York, (2000).

[4] G. R. Goldstein and J. A. Goldstein, Regularity for Semilinear Abstract Cauchy Problems. Lect. Notes in Pure and Applied Maths. 178. Marcel Dekker, New York, pp. 99-105, (1996).

[5] J. K. Hale and J. Kato, Phase Space for Retarded Equations with Infinite Delay, Funkcial. Ekvac. 21, pp. 11-41, (1978).

[6] H. R. Henríquez, Regularity of Solutions of Abstract Retarded Functional Differential Equations with Unbounded Delay. Nonlinear Analysis, Theory, Methods $\&$ Applications 28 (3), pp. 513531, (1997).

[7] H. R. Henríquez, Periodic Solutions of Quasi-Linear Partial Functional Differential Equations with Unbounded Delay. Funkcialaj Ekvac. 37 (2), pp. 329-343, (1994).

[8] Henríquez, H. R., Approximation of Abstract Functional Differential Equations with Unbounded Delay, Indian J. Pure and Applied Maths. 27 (4), pp. 357-386, (1996).

[9] Y. Hino, S. Murakami and T. Naito, Functional Differential Equations with Infinite Delay. Lect. Notes in Maths. 1473. SpringerVerlag, Berlin, (1991). 
[10] C. M. Marle, Mesures et Probabilités. Hermann, Paris, (1974).

[11] A. Pazy, Semigroups of Linear Operators and Applications to Partial Differential Equations. Springer-Verlag, New York, (1983).

[12] W. Ruess, Existence and Stability of Solutions to Partial Functional Differential Equations with Delay. Adv. Diff. Eqns. 4 (6), pp. 843-876, (1999).

[13] W. Ruess, Existence of Solutions to Partial Functional Differential Equations with Delay. in A. G. Kartsatos (ed), Theory and Applications of Nonliner Operators of accretive and Monotone Type. Lect. Notes in Pure and Applied Maths. 178, M. Dekker, New York, 1996, pp.259-288.

[14] C. C. Travis and G. F. Webb, Existence and Stability for Partial Functional Differential Equations, Trans. Amer. Math. Soc. 200, pp. 395-418, (1974).

Received : June, 2002.

\section{HERNÁN R. HENRÍQUEZ}

Departamento de Matemática

Universidad de Santiago de Chile

Casilla 307

Correo 2

Santiago

Chile

e-mail : hhenriqu@lauca.usach.cl 